\title{
Prognostic significance of macrophage invasion in hilar cholangiocarcinoma
}

\author{
Georgi Atanasov ${ }^{1 * \dagger}$, Hans-Michael Hau ${ }^{2^{*}+}$, Corinna Dietel ${ }^{2}$, Christian Benzing ${ }^{1}$, Felix Krenzien ${ }^{1}$, Andreas Brandl ${ }^{1}$, \\ Georg Wiltberger², Ivan Matia², Isabel Prager², Katrin Schierle³, Simon C. Robson ${ }^{4}$, Anja Reutzel-Selke ${ }^{1}$, \\ Johann Pratschke ${ }^{1}$, Moritz Schmelzle $e^{1,5^{*}}$ and Sven Jonas ${ }^{6^{*}}$
}

\begin{abstract}
Background: Tumor-associated macrophages (TAMs) promote tumor progression and have an effect on survival in human cancer. However, little is known regarding their influence on tumor progression and prognosis in human hilar cholangiocarcinoma.

Methods: We analyzed surgically resected tumor specimens of hilar cholangiocarcinoma $(n=47)$ for distribution and localization of TAMs, as defined by expression of CD68. Abundance of TAMs was correlated with clinicopathologic characteristics, tumor recurrence and patients' survival. Statistical analysis was performed using SPSS software.

Results: Patients with high density of TAMs in tumor invasive front (TIF) showed significantly higher local and overall tumor recurrence (both $\rho<0.05$ ). Furthermore, high density of TAMs was associated with decreased overall (one-year $83.6 \%$ vs. $75.1 \%$; three-year $61.3 \%$ vs. $42.4 \%$; both $\rho<0.05$ ) and recurrence-free survival (one-year $93.9 \%$ vs. $57.4 \%$; three-year $59.8 \%$ vs. $26.2 \%$; both $\rho<0.05$ ). TAMs in TIF and tumor recurrence, were confirmed as the only independent prognostic variables in the multivariate survival analysis (all $\rho<0.05$ ).

Conclusions: Overall survival and recurrence free survival of patients with hilar cholangiocarcinoma significantly improved in patients with low levels of TAMs in the area of TIF, when compared to those with a high density of TAMs. These observations suggest their utilization as valuable prognostic markers in routine histopathologic evaluation, and might indicate future therapeutic approaches by targeting TAMs.
\end{abstract}

Keywords: Hilar cholangiocarcinoma, Tumor associated macrophages, TAMs, CD68, Liver resection

\section{Background}

Hilar cholangiocarcinoma represents the most common cancer arising within the extrahepatic bilary tree and extended liver resection or liver transplantation following a highly selective protocol with combined neoadjuvant radiochemotherapy represent the only curative treatment [1]. High risk of tumor recurrence remains a serious

\footnotetext{
* Correspondence: georgi.atanasov@charite.de; hansmichael.hau@medizin. uni-leipzig.de; moritz.schmelzle@charite.de; svnjns@gmail.com

Moritz Schmelzle and Sven Jonas are co-senior authors.

${ }^{\dagger}$ Equal contributors

'Department of General, Visceral and Transplantation Surgery and Department of General, Visceral, Vascular and Thoracic Surgery, Charité Universitätsmedizin Berlin, Charitéplatz 1, 10117 Berlin, Germany

${ }^{2}$ Department of Visceral-, Transplantation-, Thoracic- and Vascular Surgery,

University Hospital Leipzig, Leipzig, Germany

${ }^{6}$ Centre Hépato-Biliaire, Université Paris Sud, Hôpital Paul Brousse, Paris, France

Full list of author information is available at the end of the article
}

problem, even if liver resection is combined with extrahepatic hilar en bloc resection [2,3]. The seventh edition of the TNM classification separates extrahepatic bile duct tumors into perihilar and distal tumors [4]. Modifications of staging systems for hilar cholangiocarcinoma in order to enhance prognostic accuracy have recently been proposed $[5,6]$. There is an urgent need to identify prognostic markers associated with recurrence and survival. A better understanding of underlying biological mechanisms might further help to improve treatment options in this tumor entity.

All classes of leukocytes are found within malignant tumors. Tumor-associated macrophages (TAMs) constitute up to $50 \%$ of this leukocyte cell population. Monocytes are recruited from the circulation at sites of injury, inflammation, infection, and malignancy, where they differentiate into tissue macrophages [7-10]. TAMs are diffusely found 
throughout tumorous tissue in localized zones, e.g. tumor invasive fronts (TIF), around ductal and in tumor stromal areas $[11,12]$. Experimental data have highlighted a fundamental role of TAMs in tumor progression [13]. High abundance of TAMs is associated with an unfavorable prognosis in hepatocellular carcinoma (HCC), esophageal, ovarian and breast cancer and recent studies have emphasized a link between their abundance in tumor tissues and the process of tumor spread [14-19].

The clinical significance of infiltrating TAMs remains uncertain in hilar cholangiocarcinoma. The aim of this study was therefore to evaluate the relationship between abundance of TAMs and a presumed association with tumor growth, metastasis, recurrence and clinical prognosis in hilar cholangiocarcinoma.

\section{Methods}

\section{Patients and tumor samples}

A total of 47 patients who underwent major hepatectomy between January 1996 and December 2002 for hilar cholangiocarcinoma were included in the study. Hilar cholangiocarcinoma was confirmed histopathologically and classified according to the American Joint Committee on Cancer/Union Internationale Contra Cancrum tumornode-metastasis classification (UICC) classification. Written informed consent was obtained from all patients. This study was approved by the ethics committee of Charite Universitätsmedizin Berlin.

In all patients liver resection was in curative intent. None of the patients received neoadjuvant radio- and/or chemotherapy prior to surgery. None of the patients died in the postoperative course. In 37 of $47(78.7 \%)$ patients a curative resection was accomplished (histopathologically confirmed negative resection margin; R0 status), in 5 patients $(10.6 \%) \mathrm{R} 1$ status was diagnosed and in another 5 (10.6\%) patients R2 situation was pathologically confirmed.

Formalin-fixed, paraffin-embedded tumor samples were retrieved from the files of the Institute of Pathology. Tissue blocks embedding a representative sample of the tumor were used. Histological diagnosis of the primary tumor stage and nodal status were determined by hematoxylin and eosin (H\&E) stained sections. The clinicopathological characteristics of the study population are depicted in Table 1.

\section{Immunohistochemistry}

Formalin-fixed and paraffin-embedded tumor sections (5 $\mu \mathrm{m}$ thick) were dewaxed and rehydrated. Antigen retrieval was performed by heating the slides in $10 \mathrm{mM}$ Tris buffer with $1 \mathrm{mM}$ EDTA (pH 9) in a streamer for $20 \mathrm{~min}$. Endogenous peroxidase activity was inhibited with $3 \% \mathrm{H}_{2} \mathrm{O}_{2}$ for $5 \mathrm{~min}$. After washing with Tris buffered saline (TBS) with tween, the endogenous biotin was
Table 1 Clinicopathological characteristics of the patients included in the study

\begin{tabular}{|c|c|}
\hline \multicolumn{2}{|c|}{ Clinicopathological characteristics } \\
\hline Variable & Value (\%) \\
\hline No. of patients & 47 \\
\hline \multicolumn{2}{|l|}{ Gender } \\
\hline Male & $23(48.9 \%)$ \\
\hline Female & $24(51.1 \%)$ \\
\hline \multicolumn{2}{|l|}{ Patient age } \\
\hline$\leq 60$ & $23(48.9 \%)$ \\
\hline$>60$ & $24(51.1 \%)$ \\
\hline \multicolumn{2}{|c|}{ Histologic differentiation } \\
\hline Well/Moderate & $40(85.1 \%)$ \\
\hline Poor & $7(14.9 \%)$ \\
\hline \multicolumn{2}{|l|}{ Pathologic T stage } \\
\hline $\mathrm{T} 2$ & $16(34.0 \%)$ \\
\hline T3 & $31(66.0 \%)$ \\
\hline \multicolumn{2}{|l|}{ Pathologic N stage } \\
\hline Negative & $32(69.1 \%)$ \\
\hline Positive & $15(31.9 \%)$ \\
\hline \multicolumn{2}{|c|}{ Perineural sheath infiltration } \\
\hline Negative & $4(8.5 \%)$ \\
\hline Positive & $43(91.5 \%)$ \\
\hline \multicolumn{2}{|c|}{ Lymphangiosis carcinomatosa } \\
\hline Negative & $25(53.2 \%)$ \\
\hline Positive & $22(46.8 \%)$ \\
\hline \multicolumn{2}{|c|}{ Perivascular lymphangiosis } \\
\hline Negative & $24(51.1 \%)$ \\
\hline Positive & $23(48.9 \%)$ \\
\hline \multicolumn{2}{|l|}{ Vascular invasion } \\
\hline Negative & $32(68.1 \%)$ \\
\hline Positive & $15(31.9 \%)$ \\
\hline \multicolumn{2}{|l|}{ Tumor recurrence } \\
\hline Without & $22(46.8 \%)$ \\
\hline With & $25(53.2 \%)$ \\
\hline \multicolumn{2}{|l|}{ Local recurrence } \\
\hline Without & $27(57.4 \%)$ \\
\hline With & $20(42.6 \%)$ \\
\hline \multicolumn{2}{|l|}{ Distant metastases } \\
\hline Without & $36(76.6 \%)$ \\
\hline With & 11 (23.4\%) \\
\hline
\end{tabular}

suppressed by sequential incubations with $0,1 \%$ avidin and $0,01 \%$ biotin (Dako, Glostrup, Denmark) for $10 \mathrm{~min}$ each at room temperature. Additional nonspecific binding sites were blocked with $3 \%$ skimmed milk powder for $30 \mathrm{~min}$ at room temperature. Tissue sections were 
incubated with the monoclonal mouse antibody antihuman CD68 Clone PG-M1 (1:50, Dako, Glostrup, Denmark) for $30 \mathrm{~min}$ at room temperature. The universal LSAB+ system-HRP (Dako, Glostrup, Denmark) and the $\mathrm{DAB}+$ liquid substrate chromogen system (Dako, Glostrup, Denmark) was applied for the visualization of the antibody reaction. The biotinylated anti-rabbit, anti-mouse and antigoat immunoglobulin and the streptavidin conjugated horseradish peroxidase of the universal LSAB+ system was incubated consecutively for $20 \mathrm{~min}$ each. Sections were counterstained with hematoxylin. Specificity controls were performed without primary antibody.

\section{Quantification of CD68 density}

All specimens were evaluated by three independent researchers (GA, CD and IP), and an independent pathologists (KS), without any knowledge of prognosis or clinicopathological variables. TAMs were defined by their expression of CD68. For quantification of infiltrating TAMs the whole tumor area was thoroughly investigated for presence of CD68-positive cells. The evaluation of TAMs density was performed as percent immunohistochemical CD68 staining related to tumor cell amount.

The density of TAMs in the whole tumor area (tumorous tissue, TT) was semi-quantitatively classified into the following categories: 0 , negative; $1,1-25 \% ; 2,26-75 \%$; and $3,>75 \%$. For statistical analysis, TT scores 0 and 1 were categorized as low density, and TT scores 2 and 3 as high density of TAMs. The density of TAMs in the tumor infiltrating front (TIF) were semi-quantitatively classified into the following major categories: 0 , negative; $1,1-25 \%$ and 2, $>25 \%$. For statistical analysis, TIF scores 0 and 1 were categorized as low density or negative, TIF score 2 as high density (or positive).

Patients with hilar cholangiocarcinoma were divided into several groups either by 'low' or 'high' density of macrophages in tumorous tissue ('low TT CD68 group'; $n=24$ ) and 'high TT CD68 group'; $n=23$, respectively), or 'low' or 'high' density of TAMs in TIF ('low TIF CD68 group' $n=21$; and 'high TIF CD68 group', $n=26$, respectively).

\section{Statistical analysis}

Survival analysis, univariate analysis and Kaplan-Meier curves were generated with assistance of the SPSS software program (Version 19.0.0 / Year 2010). Comparison of categorical and continuous variables was performed using the $\mathrm{Chi}^{2}$-test and the Wilcoxon-test, respectively. Survival data were compared with the log rank-test. Variables with a significant influence on survival in the univariate analysis were entered into a cox regression analysis. A difference was considered significant for $p<0.05$.

\section{Results}

The median follow-up after resection in all patients was 28 months (range 2.3 to 105.7 months). The overall 1-, 3- and 5-year survival of our cohort was 78.8, 51.4 and $31.7 \%$, respectively (Fig. 1a). The overall $1-, 3$ - and 5 year recurrence-free survival rates were $68.8,40.1$ and $31.3 \%$, respectively (Fig. 1e). 27 (57.4\%) of 47 patients died within the follow-up interval. 25 patients (53.2\%) developed tumor recurrence and $24(51.0 \%)$ patients died for recurrence of hilar cholangiocarcinoma. Local tumor recurrence was seen in 20 (42.6\%) patients, while $5(10.6 \%)$ patients developed distant metastases without manifestation of local recurrence (Table 1). In 6 (12.7\%) patients with local recurrence of hilar cholangiocarcinoma a metastatic spread of the tumor to distant sites was detected. 3 (6.3\%) patients died without evidence for tumor-related cause and had no signs of tumor recurrence at the time of death.

\section{Macrophage distribution in hilar cholangiocarcinoma}

TAMs showed a vast homogenous distribution in tumor stroma and tumor parenchyma, TIF and perivascular areas, respectively (Fig. 2a-c). No clusters or 'clumping' of TAMs in tumor tissue has been observed. The infiltration patterns did not reveal any preference for the tumor perivascular areas. The majority of TAMs were apparently located in direct contact with or adjacent to tumor cells. Interestingly, a high density of TAMs was observed at TIF in 26 patients (TIF score $2 /$ high density/positive), while rest of tumor specimens $(n=21)$ displayed only a scarce or complete absence of infiltrating TAMs (TIF scores 0 and $1 /$ low density/negative) (Fig. 2c).

\section{The presence of tumor-infiltrating CD68-positive} macrophages in tumor invasive front associates with tumor recurrence in patients with hilar cholangiocarcinoma High abundance of macrophages in TIF correlated with a significantly increased overall tumor recurrence. 18 (69.2 \%) of 26 patients in high TIF CD68 group displayed tumor recurrence $(\rho=0.015)$. In the low TIF CD68 group 7 (33.3\%) of 21 patients had recurrent disease. Moreover, patients with high levels of macrophages in TIF showed a significantly enhanced incidence of local tumor recurrence $(\rho=0.0001)$.

17 (65.4 \%) of 26 patients in the high TIF CD68 group suffered a local tumor recurrence, whereas 3 (14.3\%) out of 21 patients in the low TIF CD68 group did not show local tumor recurrence (Table 2). After R0 resection $(n=37)$, a low CD68 abundance in TIF ('R0 low CD68 TIF group'; $n=16)$ correlated with a significantly lower rate of disease recurrence, as well. 11 (68.8\%) and 15 (93.8\%) patients in the low CD68 TIF group did not develop overall or local tumor recurrence following R0 surgery ( $\rho=0.064$ and $\rho=0.001$, respectively). 


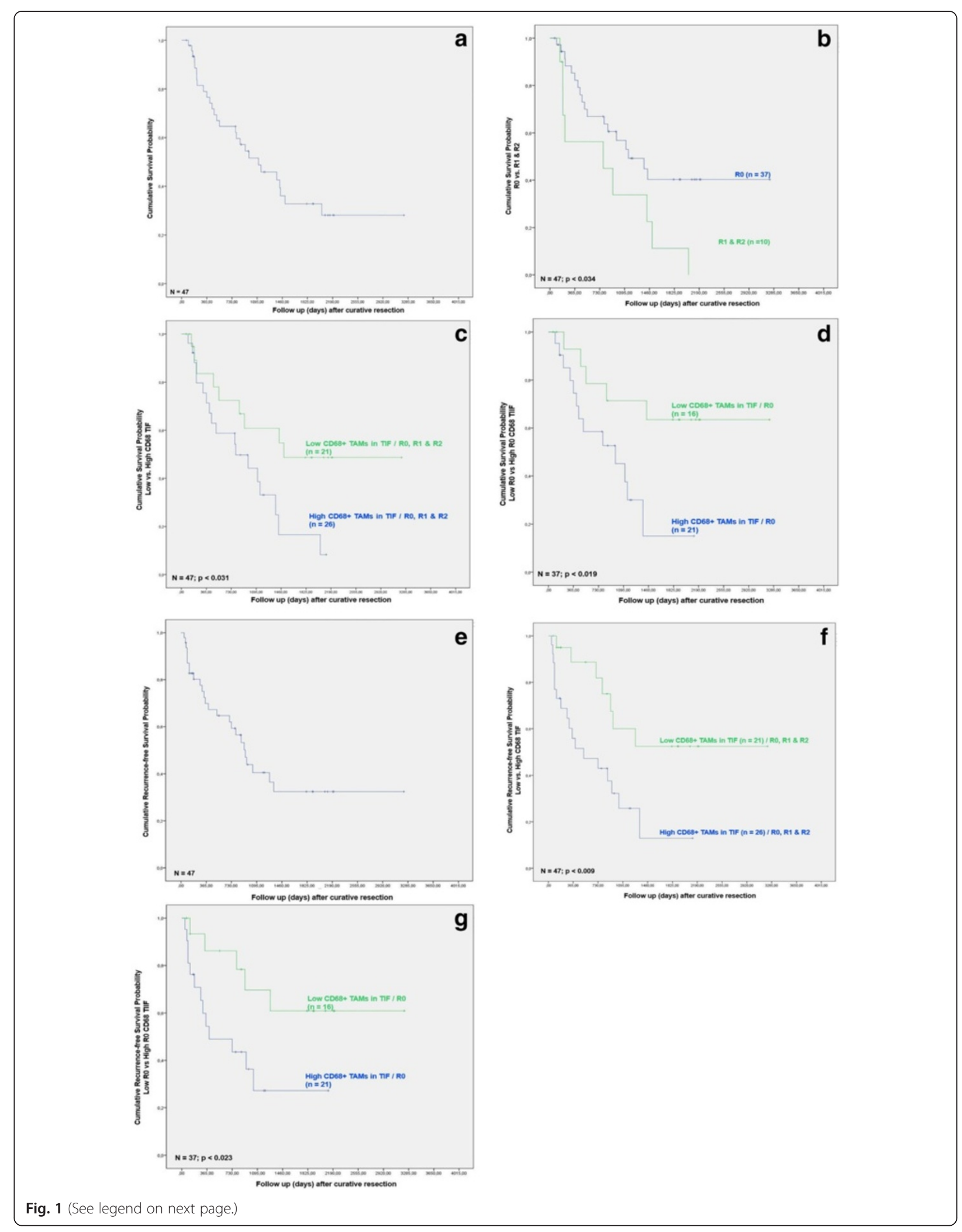


(See figure on previous page.)

Fig. 1 a Overall survival after surgery for hilar cholangiocarcinoma $(n=47)$. b Overall survival after surgery for hilar cholangiocarcinoma $(n=37)$ according to R status. c Overall survival after surgery referred to CD68-positve TAMs in the tumor infiltration front (TIF) regardless of $\mathrm{R}$ status ( $n=47$ ). d Overall survival after surgery referred to TAMs in the tumor infiltration front (TIF) after R0 resection ( $n=37$ ). e Recurrence-free survival of all patients after surgery for hilar cholangiocarcinoma $(n=47)$. $\mathbf{f}$ Recurrence-free survival after surgery correlated to TAMs in the tumor invasive front (TIF) regardless of R status ( $n=47)$. $\mathbf{g}$ Recurrence-free survival correlated to TAMs in the tumor invasive front (TIF) following R0 surgery $(n=37)$

Other well established common prognostic factors were assessed, as well. 5 (19.2\%) of 26 patients in high TIF CD68 group had poor histologic tumor differentiation. In the low TIF CD68 group 2 (9.5\%) of 21 patients displayed poor histologic differentiation $(\rho=0.307)$. Related to angioinvasion, $8(30.8 \%)$ of 26 and 7 (33.3\%) of 21 patients in the high or low CD68 TIF group, respectively, showed a microscopic angioinvasion $(\rho=0.549) .18(69.2 \%)$ of 26 and $13(61.9 \%)$ of 21 patients in the high or low CD68 TIF group, respectively, showed a more advanced T stage $(\rho=0.413)$. Related to perineural sheet infiltration, 3 (11.5\%) of 26 and 1 (4.8\%) of 21 patients in the high or low CD68 TIF group, respectively, showed an absence of perineural sheet infiltration $(\rho=0.390)$.

\section{Prognostic significance of CD68-positive macrophages in hilar cholangiocarcinoma}

Next, we analyzed whether TAMs in addition to other clinicopathological parameters predict survival after resection for hilar cholangiocarcinoma. In the univariate analysis $\mathrm{R}$ category, overall and local tumor recurrence, and low density of macrophages in TIF were associated with a statistically significant improvement of patient survival after resection $(\rho=0.039, \rho=0.001, \rho=0.001$ and $\rho=0.036$, respectively). As related to recurrence-free survival, in univariate analysis only low macrophages in TIF $(\rho=0.013)$ were associated with a statistically significant improvement.

In multivariate analysis presence of TAMs in TIF (related to recurrence-free survival for all patients, as well for R0 status only) and tumor recurrence were
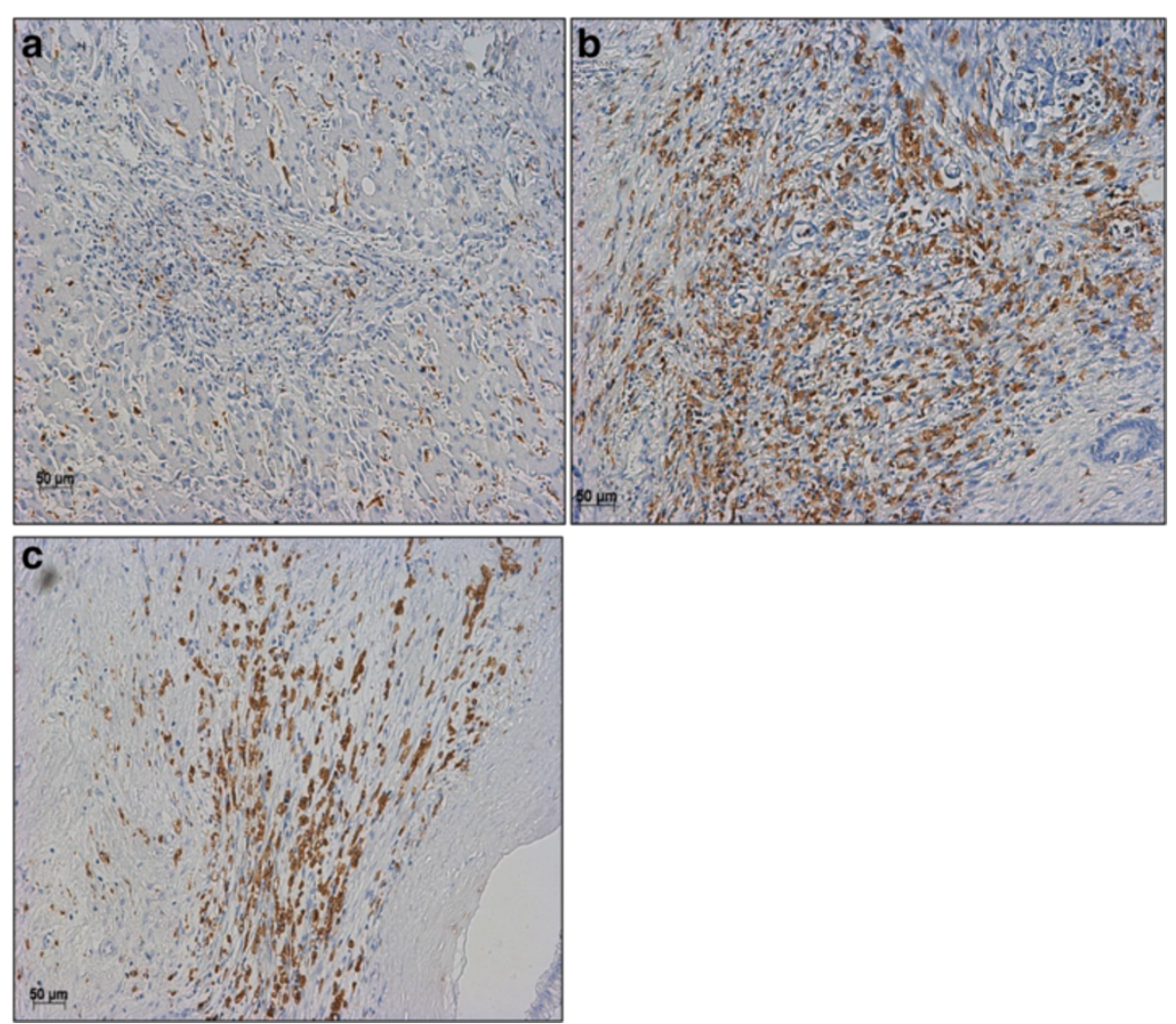

Fig. 2 a CD68-positive macrophages in normal liver tissue. Normal liver parenchyma revealed a homogenous infiltration pattern with no preference for perivascular areas or clumping of TAMs. Original magnification: x 200; scale bar = $50 \mu$ m. b Hilar cholangiocarcinoma stained with mAb CD68 PG-M1 with a high abundance of TAMs (tumorous tissue "TT" score 3). Original magnification: x 200; scale bar = 50 $\mu$ m. c Tumor infiltration front (TIF) positive for CD68-positive TAMs (TIF score 2 / high abundance / positive). Original magnification: $\times 200$; scale bar = 50 $\mu$ m 
Table 2 Correlation of CD68-positiveTAMs in the tumor invasive front with clinicopathological characteristics of hilar cholangiocarcinoma

\begin{tabular}{|c|c|c|c|}
\hline \multicolumn{4}{|l|}{ Clinicopathological analysis } \\
\hline Variable & high CD68 & low CD68 & $\mathrm{p}$ \\
\hline No. of patients & 26 & 21 & \\
\hline Gender & & & 0.552 \\
\hline Male & $13(50 \%)$ & $10(47.6 \%)$ & \\
\hline Female & $13(50 \%)$ & $11(52.4 \%)$ & \\
\hline Patient age, years & & & 0.448 \\
\hline$\leq 60$ & $14(53.8 \%)$ & $12(47.6 \%)$ & \\
\hline$>60$ & $12(46.2 \%)$ & $11(52.4 \%)$ & \\
\hline Histological differentiation & & & 0.307 \\
\hline Well/moderate & $21(80.8 \%)$ & $19(90.5 \%)$ & \\
\hline Poor & $5(19.2 \%)$ & $2(9.5 \%)$ & \\
\hline Pathological T stage & & & 0.413 \\
\hline $\mathrm{T} 2$ & $8(30.8 \%)$ & $8(38.1 \%)$ & \\
\hline T3 & 18 (67.9\%) & $13(61.9 \%)$ & \\
\hline Pathological N stage & & & 0.307 \\
\hline Negative & 19 (73.1 \%) & $13(61.9 \%)$ & \\
\hline Positive & $7(26.9 \%)$ & $8(38.1 \%)$ & \\
\hline Perineural sheath infiltration & & & 0.390 \\
\hline Negative & $3(11.5 \%)$ & 1 (4.8\%) & \\
\hline Positive & $23(88.5 \%)$ & $20(95.2 \%)$ & \\
\hline Lymphangiosis carcinomatosa & & & 0.576 \\
\hline Negative & $12(46.2 \%)$ & $10(47.6 \%)$ & \\
\hline Positive & $14(53.8 \%)$ & $11(52.4 \%)$ & \\
\hline Perivascular lymphangiosis & & & 0.552 \\
\hline Negative & $13(50 \%)$ & $11(52.4 \%)$ & \\
\hline Positive & $13(50 \%)$ & $10(47.6 \%)$ & \\
\hline Vascular invasion & & & 0.549 \\
\hline Negative & $18(69.2 \%)$ & $14(66.6 \%)$ & \\
\hline Positive & $8(30.8 \%)$ & $7(33.3 \%)$ & \\
\hline Tumor recurrence & & & 0.015 \\
\hline Without & $8(30.8 \%)$ & $14(66.7 \%)$ & \\
\hline With & 18 (69.2 \%) & 7 (33.3 \%) & \\
\hline Local recurrence & & & 0.001 \\
\hline Without & $9(34.6 \%)$ & $18(85.7 \%)$ & \\
\hline With & 17 (65.4 \%) & $3(14.3 \%)$ & \\
\hline Distant metastases & & & 0.390 \\
\hline Without & 19 (73.1 \%) & $17(81.7 \%)$ & \\
\hline With & 7 (26.9 \%) & $4(19.0 \%)$ & \\
\hline
\end{tabular}

identified as independent prognostic factors for survival (all $\rho<0.05$; Table 3). Other well established common prognostic factors, e. g. T stage and angioinvasion, did not exert prognostic significance in the current work.
Influence of macrophages on overall and recurrence-free survival in hilar cholangiocarcinoma

Based on the identification of TAMs in TIF as an independent prognostic factor in both the univariate and multivariate analysis, we investigated its effect on patients' survival in hilar cholangiocarcinoma. The presence of TAMs in TIF affected patients' survival after resection for hilar cholangiocarcinoma. Univariate analysis revealed a significantly better survival in the low TIF CD68 group $(\rho=0.013)$. The overall survival rates were 83.6 and $61.3 \%$ at 1 - and 3-year post surgery in comparison with 75.1 and $42.4 \%$ at 1 - and 3-year following resection for patients with low and high abundance of CD68-positive macrophages in TIF, respectively (Fig. 1c). Moreover, a low density of CD68-positive cells in TIF correlated with a significantly improved recurrence free survival. The 1-, 3-, and 5-year recurrence-free survival rates of patients with tumors that displayed low density of CD68-positive cells in TIF were 93.9, 59.8 and $51.8 \%$, whereas the 1-, 3-, and 5-year recurrence-free survival rates of patients with tumors with a high abundance of CD68-positive cells in TIF were 57.4, 26.2 and $12.1 \%$, respectively (Fig. 1f). The difference was statistically significant $(p=0.009)$. Following $\mathrm{R} 0$ resection, a low abundance of CD68-positive cells in TIF correlated with an improved overall and recurrence-free survival, as well ( $p=0.19$ and $p=0.023$, respectively) (Fig. $1 \mathrm{~b}, \mathrm{~d}, \mathrm{~g}$ ).

Histologic differentiation and perineural sheet infiltration showed a distinct trend towards an improved overall survival, though without reaching statistical significance ( $\rho=0.103$ and $\rho=0.224$, respectively). Overall survival and recurrence free survival of patients with hilar cholangiocarcinoma were significantly better in patients with low levels of TAMs in the area of TIF when compared to those with a high density of TAMs.

\section{Discussion}

Analyzing immunoreactivity for CD68-positive TAMs in tumor samples from patients who underwent resection for hilar cholangiocarcinoma, we were able to show that (1.) a high abundance of CD68 in TIF is a risk factor for local recurrence and overall tumor recurrence, (2.) serves as an independent prognostic factor for survival, and (3.) translates into dismal overall as well as recurrence-free survival rates.

High density of TAMs in TIF was an independent negative prognostic factor for recurrence-free survival. Espinosa et al. demonstrated TAMs to correlate with increased microvessel proliferation and enhanced tumor angiogenesis [20]. Thus, reduced density of CD68 in TIF might mitigate tumor angiogenesis and improve survival in hilar cholangiocarcinoma. In the univariate analysis a negative-margin (R0) tumor resection correlated significantly with improved survival; however, this could not 
Table 3 Univariate and multivariate analysis of prognostic factors in patients with hilar cholangiocarcinoma

\begin{tabular}{|c|c|c|c|c|}
\hline Variable & Category & Odds ratio & $p$ & Confidence interval \\
\hline \multicolumn{5}{|c|}{ Univariate analysis } \\
\hline Age & $\leq 60 />60$ & 1.588 & 0.239 & $0.735-3.430$ \\
\hline Gender & male/female & 1.287 & 0.517 & $0.600-2.761$ \\
\hline perivascular lymphangiosis & negative/positive & 0.734 & 0.430 & $0.340-1.583$ \\
\hline R-Classification & negative/positive & 2.325 & 0.039 & $1.042-5.189$ \\
\hline Histologic differentiation & well or moderate/poor & 2.141 & 0.103 & $0.857-5.344$ \\
\hline Vascular invasion & negative/positive & 1.285 & 0.569 & $0.542-3.045$ \\
\hline pT & $\mathrm{pT} 2 / \mathrm{pT} 3$ & 1.242 & 0.597 & $0.557-2.768$ \\
\hline $\mathrm{pN}$ & negative/positive & 0.535 & 0.124 & $0.242-1.186$ \\
\hline Perineural sheet infiltration & negative/positive & 0.289 & 0.224 & $0.039-2.137$ \\
\hline Lymphangiosis carcinomatosa & negative/positive & 0.835 & 0.651 & $0.381-1.827$ \\
\hline CD68 + TAMs & high/low & 0.977 & 0.951 & $0.457-2.088$ \\
\hline CD68 + TAMs in TIF & high/low & 0.322 & 0.013 & $0.132-0.784$ \\
\hline Overall Tumor Recurrence & negative/positive & 0.112 & 0.001 & $0.033-0.37$ \\
\hline Distant Metastasis & negative/positive & 0.528 & 0.111 & $0.240-1.159$ \\
\hline Local Tumor Recurrence & negative/positive & 0.183 & 0.001 & $0.076-0.441$ \\
\hline \multicolumn{5}{|c|}{ Multivariate analysis } \\
\hline R Classification & negative/positive & 0.830 & 0.710 & $0.312-2.212$ \\
\hline Overall Tumor Recurrence & negative/positive & 0.172 & 0.024 & $0.037-0.794$ \\
\hline Local Tumor Recurrence & negative/positive & 0.544 & 0.432 & $0.119-2.486$ \\
\hline CD68-positive TAMs in TIF & high/low & 0.856 & 0.780 & $0.287-2.553$ \\
\hline \multicolumn{5}{|c|}{ Multivariate analysis (Recurrence free survival) } \\
\hline \multicolumn{5}{|c|}{$\mathrm{R} 0 \& \mathrm{R} 1 / 2$ surgery $/ n=47$} \\
\hline R Classification & negative/positive & 1.935 & 0.149 & $0.789-4.743$ \\
\hline CD68-positive TAMs in TIF & high/low & 0.324 & 0.014 & $0.132-0.796$ \\
\hline \multicolumn{5}{|c|}{$\mathrm{R} 0 \& \mathrm{R} 1 / 2$ surgery $/ n=47$} \\
\hline \multicolumn{5}{|c|}{ only R0 surgery $/ n=37$} \\
\hline Histologic Differentiation & well or moderate/poor & 2.549 & 0.109 & $0.811-8.012$ \\
\hline CD68-positive TAMs in TIF & high/low & 0.286 & 0.024 & $0.097-0.846$ \\
\hline only R0 surgery $/ n=37$ & & & & \\
\hline
\end{tabular}

be confirmed in the multivariate analysis which is likely to be due to the small number of patients with positivemargin (R1, R2). The clinical characteristics and tumor specimens of the presented work were retrieved from a high volume center patient cohort with clinicopathologic features resembling other studies $[21,22]$. In the multivariate analysis, only tumor recurrence and high abundance of CD68 in TIF irrespective of R-status proved to be independent prognostic indicators. A high abundance of CD68 in TIF was correlated not only with a higher risk of overall tumor recurrence but also with local tumor recurrence. These variables are well established indicators for unfavorable patient outcome and poor long term survival in hilar cholangiocarcinoma. Hereby, the stronger influence of TAMs in TIF on patient survival and recurrence-free survival compared to $\mathrm{R}$ classification may be caused by correlation with multiple unfavorable clinicopathologic characteristics in hilar cholangiocarcinoma.

The tumor mass is a multifaceted playground, where different cell types, including cancer cells, fibroblasts, endothelial and immune-competent cells are entangled into a constant interaction. Paradoxically, in the majority of 
human solid cancers, cells of the innate immune system, especially TAMs, were shown to favor tumor progression by, i.e., fostering metastasis and suppressing adaptive immunity. Yet, the lack of routine implementation of the experimental immunologic rationales as diagnostic or therapeutic concepts on a clinic daily basis is striking $[23,24]$. In the present work we propose a well described and ubiquitous type of TAMs, in particular cells expressing CD68, as simple diagnostic tools prognosticating patient outcome in hilar cholangiocarcinoma.

In the current work TAMS in TIF were associated with survival and recurrence free survival and were identified as independent prognosticator. A rational clinical translation of these results suggests standardized utilization of TAMs as prognostic markers in the scope of pathological evaluation of resected specimens from patients suffering from hilar cholangiocarcinoma. This could be performed routinely in the scope of microscopical histological tumor evaluation as well as in standardized and reliable protocols on paraffin-embedded tumor specimens after staining with commercially available and commonly used antibodies against the macrophage marker CD68.

Another possibility for clinical utilization is the targeting of TAMs in tumors. The pro-tumor features of TAMs render these cell types attractive targets for biological anti-cancer therapies. To date, tumor progression is successfully treated by depletion of TAMs in preclinical animal models. Moreover, the combination of macrophage depleting agents with sorafenib, a potent inhibitor of tyrosine protein kinases (e.g. VEGF and platelet-derived growth factor receptor (PDGRF)), enhances significantly the efficacy of sorafenib alone in a xenograft model of hepatocellular carcinoma [25].

High recurrence rates after surgical resection for hilar cholangiocarcinoma imply an urgent need for reliable prognostic markers aiding the selection of appropriate patients who will benefit from radical surgical strategies. In particular, suitable patients for liver transplantation need to be cautiously selected, on grounds of a common donor organ shortage. CD68-positive TAMs and tumor necrosis may prove as valuable tools regarding patient selection. Emerging experimental studies reveal a potential role for selective in vivo visualization of circulating monocytes/macrophages and TAMs by magnetic resonance imaging (MRI) techniques allowing 3D spatial resolution up to $50 \mu \mathrm{m}$ [26-31]. With a further development and clinical implementation of these imaging tools the evaluation of TAMs could offer an additional diagnostic modality for the patient selection for further treatment strategies.

However, drawbacks in the utilization of CD68 as a selection criterion prior surgery are to be considered, as well. To date, reliable clinical evaluation of these markers is only possible in resected tumor specimens following surgery. It should be also considered that tumor biopsies prior surgery will not provide representative tissue specimens and will not conform to the principles of a surgical approach using a 'no-touch technique' [32].

\section{Conclusions}

In conclusion, in the current study we demonstrate for the first time that presence of CD68-positive TAMs in tumor invasive front correlates with tumor recurrence and serves as an independent prognostic factor for survival in hilar cholangiocarcinoma. Thus, TAMs may be crucially involved in the progression of this tumor entity and their utilization as a diagnostic tool or targeting TAMs, may deliver new therapeutic approaches in this cancer.

\section{Abbreviations}

HCC: Hepatocellular carcinoma; H\&E: Hematoxylin and eosin; mAb: Monoclonal antibody; PDGRF: Platelet-derived growth factor receptor; TBS: Tris buffered saline; TAMs: Tumor associated macrophages; TIF: Tumor infiltrating front; UICC: Union Internationale Contra Cancrum; VEGF: Vascular endothelial growth factor.

\section{Competing interests}

The authors declare that they have no competing interests.

\section{Authors' contributions}

All authors have read and approved the manuscript, and ensure that this is the case. GA prepared and wrote the manuscript, developed and organized the project, analyzed the data, performed experimental procedures and statistical analysis; H.M. H participated in analyzing the data, preparing, reviewing and proofreading the article; CD participated in conducting the experimental procedures, analyzing the data, and preparing, reviewing and proofreading the article; IP participated in conducting the immunological analysis, analyzing the data and reviewing and proofreading the article; JP, GW, FK, IM and CB contributed to reviewing and proofreading the article, and organizing the data. $A B$ and $S C R$ contributed to reviewing and proofreading the article; KS contributed to reviewing, proofreading the article, supervised patho-histological data assessment and performed pathological evaluation of tumor specimens; AR-S contributed to reviewing, proofreading the article and conducting statistical analysis; MS participated in developing, organizing and analyzing the data; and writing, reviewing, and proofreading the article; SJ contributed to acquiring and analyzing the data; and writing, reviewing, and proofreading the article.

\section{Authors' information}

Georgi Atanasov and Hans-Michael Hau contributed equally and share authorship. Moritz Schmelzle and Sven Jonas are co-senior authors.

\section{Acknowledgements}

The authors are most grateful to Ralf Henkelmann, M.D. (Department of Orthopedics, Traumatology and Plastic Surgery, University Hospital Leipzig, Leipzig, Germany) and Micaela Ebert, M.D. (Department of Rhythmology, Herzzentrum, University Hospital Leipzig, Leipzig, Germany) for the essential collegial support and creativity in the accrual of this work.

\section{Funding}

The work presented in this paper was made possible by funding from the German Federal Ministry of Education and Research (BMBF 1315883).

\section{Author details}

'Department of General, Visceral and Transplantation Surgery and Department of General, Visceral, Vascular and Thoracic Surgery, Charité Universitätsmedizin Berlin, Charitéplatz 1, 10117 Berlin, Germany. ${ }^{2}$ Department of Visceral-, Transplantation-, Thoracic- and Vascular Surgery, University Hospital Leipzig, Leipzig, Germany. ${ }^{3}$ Institute of Pathology, University Hospital Leipzig, Leipzig, Germany. ${ }^{4}$ The Transplant Institute and Division of Gastroenterology, Beth Israel Deaconess Medical Center, Harvard 
University, Boston, MA, USA. ${ }^{5}$ Translational Centre for Regenerative Medicine, Leipzig University, Leipzig, Germany. ${ }^{6}$ Centre Hépato-Biliaire, Université Paris Sud, Hôpital Paul Brousse, Paris, France.

\section{Received: 8 October 2014 Accepted: 15 October 2015}

Published online: 24 October 2015

\section{References}

1. de Groen PC, Gores GJ, LaRusso NF, Gunderson LL, Nagorney DM. Biliary tract cancers. N Engl J Med. 1999:341:1368-78.

2. Jonas S, Benckert C, Thelen A, Lopez-Hänninen E, Rösch T, Neuhaus P. Radical surgery for hilar cholangiocarcinoma. Eur J Surg Oncol. 2008;34(3):263-71.

3. Jarnagin WR, Shoup M. Surgical management of cholangiocarcinoma. Semin Liver Dis. 2004;24:189-99.

4. Juntermanns B, Sotiropoulos GC, Radunz S, Reis H, Heuer M, Baba HA, et al. Comparison of the sixth and the seventh editions of the UICC classification for perihilar cholangiocarcinoma. Ann Surg Oncol. 2013;20(1):277-84.

5. Zaydfudim VM, Clark CJ, Kendrick ML, Que FG, Reid-Lombardo KM, Donohue $\mathrm{JH}$, et al. Correlation of staging systems to survival in patients with resected hilar cholangiocarcinoma. Am J Surg. 2013;206(2):159-65.

6. Ebata T, Kosuge T, Hirano S, Unno M, Yamamoto M, Miyazaki M, et al. Proposal to modify the International Union Against Cancer staging system for perihilar cholangiocarcinomas. Br J Surg. 2014;101(2):79-88.

7. Gordon S. Alternative activation of macrophages. Nat Rev Immunol. 2003;3:23-35.

8. Sica A, Schioppa T, Mantovani A, Allavena P. Tumour-associated macrophages are a distinct $M 2$ polarised population promoting tumour progression: potential targets of anti-cancer therapy. Eur J Cancer. 2006;42:717-27.

9. Pollard JW. Tumour-educated macrophages promote tumour progression and metastasis. Nat Rev Cancer. 2004:4:71-8.

10. Hallam S, Escorcio-Correia M, Soper R, Schultheiss A, Hagemann T. Activated macrophages in the tumour microenvironment-dancing to the tune of TLR and NF-kappaB. J Pathol. 2009;219:143-52.

11. Lee $A H$, Happerfield LC, Bobrow LGand Millis RR. Angiogenesis and inflammation in invasive carcinoma of the breast. J Clin Pathol. 1997;50:669-73.

12. Goede V, Brogelli L, Ziche M, Augustin HG. Induction of inflammatory angiogenesis by monocyte chemoattractant protein-1. Int J Cancer. 1999;82:765-70.

13. Solinas G, Germano G, Montovani A, Allavena P. Tumor-associated macrophages (TAM) as major players of the cancer-related inflammation. J Leukoc Biol. 2009;86(5):1065-73.

14. An T, Sood U, Pietruk T, Cummings G, Hashimoto K, Crissman JD. In situ quantitation of inflammatory mononuclear cells in ductal infiltrating breast carcinoma. Relation to prognostic parameters. Am J Pathol. 1987;128:52-60.

15. Bingle L, Brown NJ, Lewis CE. The role of tumor-associated macrophages in tumor progression: implications for new anticancer therapies. J Pathol. 2002;196:254-65.

16. Mantovani A, Allavena P, Sica A. Tumor-associated macrophages as a prototypic type II polarized phagocyte population: role in tumor progression. Eur J Cancer. 2004;40:1660-7.

17. Tsutsui S, Yasuda K, Suzuki K, Tahara K, Higashi H, Era S. Macrophage infiltration and its prognostic implications in breast cancer: the relationship with VEGF expression and microvessel density. Oncol Rep. 2005;14:425-31.

18. Condeelis J, Pollard JW. Macrophages: obligate partners for tumor cell migration, invasion, and metastasis. Cell. 2006;124:263-6.

19. Pollard JW. Macrophages define the invasive microenvironment in breast cancer. J Leukoc Biol. 2008:84:623-30.

20. Espinosa I, José Carnicer M, Catasus L, Canet B, D'angelo E, Zannoni GF, et al. Myometrial invasion and lymph node metastasis in endometrioid carcinomas: tumor-associated macrophages, microvessel density, and HIF1A have a crucial role. Am J Surg Pathol. 2010;34(11):1708-14.

21. Aoba T, Ebata T, Yokoyama Y, Igami T, Sugawara G, Takahashi Y, et al. Assessment of nodal status for perihilar cholangiocarcinoma: location, number, or ratio of involved nodes. Ann Surg. 2013;257(4):718-25.

22. Guglielmi A, Ruzzenente A, Campagnaro T, Pachera S, Conci S, Valdegamberi $A$, et al. Prognostic significance of lymph node ratio after resection of peri-hilar cholangiocarcinoma. HPB (Oxford). 2011;13(4):240-5.

23. Shimizu K, Kotera Y, Aruga A, Takeshita N, Takasaki K, Yamamoto M. Clinical utilization of postoperative dendritic cell vaccine plus activated T-cell transfer in patients with intrahepatic cholangiocarcinoma. J Hepatobiliary Pancreat Sci. 2012;19(2):171-8.
24. Higuchi R, Yamamoto M, Hatori T, Shimizu K, Imai K, Takasaki K. Intrahepatic cholangiocarcinoma with lymph node metastasis successfully treated by immunotherapy with CD3-activated T cells and dendritic cells after surgery: report of a case. Surg Today. 2006;36(6):559-62.

25. Zhang W, Zhu XD, Sun HC, Xiong YQ, Zhuang PY, Xu HX, et al. Depletion of tumor-associated macrophages enhances the effect of sorafenib in metastatic liver cancer models by antimetastatic and antiangiogenic effects. Clin Cancer Res. 2010;16(13):3420-30.

26. Al Faraj A, Sultana Shaik A, Pureza MA, Alnafea M, Halwani R. Preferential macrophage recruitment and polarization in LPS-induced animal model for COPD: noninvasive tracking using MRI. PLoS One. 2014;9(3):e90829.

27. Al Faraj A, Luciani N, Kolosnjaj-Tabi J, Mattar E, Clement O, Wilhelm C, et al. Real-time high-resolution magnetic resonance tracking of macrophage subpopulations in a murine inflammation model: a pilot study with a commercially available cryogenic probe. Contrast Media Mol Imaging. 2013;8(2):193-203.

28. Locke LW, Mayo MW, Yoo AD, Williams MB, Berr SS. PET imaging of tumo associated macrophages using mannose coated 64Cu liposomes. Biomaterials. 2012;33(31):7785-93.

29. Jacoby C, Borg N, Heusch P, Sauter M, Bönner F, Kandolf R, et al. Visualization of immune cell infiltration in experimental viral myocarditis by (19)F MRI in vivo. MAGMA. 2014:27(1):101-6.

30. van Heeswijk RB, De Blois J, Kania G, Gonzales C, Blyszczuk P, Stuber M, et al. Selective in vivo visualization of immune-cell infiltration in a mouse model of autoimmune myocarditis by fluorine-19 cardiac magnetic resonance. Circ Cardiovasc Imaging. 2013;6(2):277-84.

31. Figueiredo S, Cutrin JC, Rizzitelli S, De Luca E, Moreira JN, Geraldes CF, et al. MRI tracking of macrophages labeled with glucan particles entrapping a water insoluble paramagnetic Gd-based agent. Mol Imaging Biol. 2013;15(3):307-15.

32. Neuhaus $P$, Thelen A, Jonas S, Puhl G, Denecke T, Veltzke-Schlieker W, et al. Oncological superiority of hilar en bloc resection for the treatment of hilar cholangiocarcinoma. Ann Surg Oncol. 2012;19(5):1602-8.

\section{Submit your next manuscript to BioMed Central and take full advantage of:}

- Convenient online submission

- Thorough peer review

- No space constraints or color figure charges

- Immediate publication on acceptance

- Inclusion in PubMed, CAS, Scopus and Google Scholar

- Research which is freely available for redistribution 